\title{
Transcriptional and functional characterization of the gene encoding acyl carrier protein in Bacillus subtilis
}

\author{
Mariano A. Martinez, Diego de Mendoza and Gustavo E. Schujman \\ Instituto de Biología Molecular y Celular de Rosario, and Departamento de Microbiología, Facultad \\ de Ciencias Bioquímicas y Farmacéuticas, Universidad Nacional de Rosario, Rosario, Argentina
}

Correspondence

Gustavo E. Schujman

schujman@ibr.gov.ar

Received 3 August 2009

Revised 2 October 2009

Accepted 21 October 2009
Acyl carrier protein (ACP) is a universal and highly conserved carrier of acyl intermediates during fatty acid biosynthesis. The molecular mechanisms of regulation of the acp $P$ structural gene, as well as the function of its gene product, are poorly characterized in Bacillus subtilis and other Gram-positive organisms. Here, we report that transcription of acp $P$ takes place from two different promoters: PfapR and PacpP. Expression of acpP from PfapR is coordinated with a cluster of genes involved in lipid synthesis (the fapR operon); the operon consists of fapR-p/s $X$ $f a b D-f a b G-a c p P$. Pacp $P$ is located immediately upstream of the acp $P$ coding sequence, and is necessary and sufficient for normal fatty acid synthesis. We also report that acp $P$ is essential for growth and differentiation, and that ACP localizes in the mother-cell compartment of the sporangium during spore formation. These results provide the first detailed characterization of the expression of the ACP-encoding gene in a Gram-positive bacterium, and highlight the importance of this protein in B. subtilis physiology.

\section{INTRODUCTION}

Acyl carrier protein (ACP) plays a central role in plant and bacterial fatty acid biosynthesis, where it shuttles the insoluble fatty acid intermediates during their synthesis and subsequent utilization by the different enzymes of the pathway. The best characterized example is Escherichia coli ACP, a 77 aa protein that has a 4-phosphopantetheine prosthetic group covalently attached to the amino acid S36 via a phosphodiester linkage (Byers \& Gong, 2007; Cronan \& Rock, 1996). During fatty acid biosynthesis, and transfer of the complete fatty acids to complex lipid precursors, the growing acyl chain is bound through a thioester linkage to the terminal thiol of the prosthetic group (Cronan \& Rock, 1996; de Mendoza et al., 2002).

In E. coli, ACP is encoded by the acpP gene, which is located within a cluster of genes involved in fatty acid biosynthesis. The gene cluster comprises (in order) plsX, $f a b H, f a b D, f a b G, a c p P$ and $f a b F$, which encode phosphate: acyl-ACP acyltransferase, $\beta$-ketoacyl-ACP synthase III, malonyl-CoA:ACP transacylase, $\beta$-ketoacyl-ACP reductase, ACP, and $\beta$-ketoacyl-ACP synthase II, respectively. acp $P$ is transcribed from two promoters: one transcript of $0.3 \mathrm{~kb}$ starts just upstream of acpP, whereas

Abbreviations: DAPI, 4,6-diamidino-2-phenylindole; RACE, rapid amplification of cDNA ends; SD, Shine-Dalgarno; tsp, transcription start point.

Details of plasmid construction, operon organization and primers used in this study are available with the online version of this paper. a longer $(1.1 \mathrm{~kb})$ transcript begins upstream of $f a b G$ (Rawlings \& Cronan, 1992). The former promoter is sufficient for the synthesis of the abundant protein ACP (Zhang \& Cronan, 1996). As expected, because of the key function of ACP in lipid biosynthesis, expression of acpP is essential in E. coli (De Lay \& Cronan, 2006).

In contrast to the wealth of knowledge available on E. coli ACP, information about this protein in Gram-positive bacteria is scarce. In Streptococcus pneumoniae, acpP is the fourth ORF in the cluster of 13 genes involved in fatty acid biosynthesis (Marrakchi et al., 2002), and it seems to be transcribed from at least two different promoters ( $\mathrm{Lu} \&$ Rock, 2006), although the physiological relevance of this has not yet been established. In Bacillus subtilis, the acpP gene is included in a cluster of lipid biosynthesis genes (Morbidoni et al., 1996). In this organism, acpP expression has been preliminarily characterized, and the results suggest the existence of more than one promoter for this gene (Morbidoni et al., 1996). Since ACP is a critical component of type II fatty acid synthase, and one of the most abundant soluble proteins in E. coli, it is of interest to study the mechanism(s) responsible for producing appropriate amounts of this protein in B. subtilis, as well the relevance of ACP in the physiology of this organism.

Here, we report that $a c p P$ is the last gene of the pentacistronic fapR operon, which encodes five proteins involved in lipid synthesis, and that acpP is transcribed from two promoters: $\mathrm{P} f a p R$ and $\mathrm{P} a c p P$. We show that $a c p P$ is co-transcribed with the upstream genes from $\mathrm{P} f a p R$, 
whereas it is specifically expressed as a single gene from the strong promoter $\mathrm{PacpP}$. Expression from the latter promoter is sufficient to supply ACP for normal fatty acid biosynthesis. We also demonstrate that, in the absence of PacpP, the synthesis of ACP becomes coupled to derepression of the fap regulon, which is composed of 10 genes involved in fatty acid and phospholipid synthesis. Finally, we present evidence that $a c p P$ is an essential gene for growth and differentiation in B. subtilis, and that ACP is preferentially confined to the mother cell during sporulation. This study provides the first physiological characterization of ACP in a Gram-positive bacterium, and describes the mechanisms employed by $B$. subtilis to control the synthesis of appropriate amounts of this essential protein.

\section{METHODS}

Strains. All bacterial strains are listed in Table 1. The B. subtilis strains were grown in Luria-Bertani (LB) broth (Difco), Spizizen minimal medium (SMM: Spizizen, 1958) or Difco sporulation medium (DSM). E. coli strains were propagated in LB broth.

Plasmids and DNA manipulation. Plasmids were constructed using standard methods, and amplified in E. coli DH5 $\alpha$ and XL1-Blue. PCR fragments were amplified from chromosomal DNA of $B$. subtilis strain JH642. For further details on DNA manipulation and plasmid construction, see Supplementary material. Plasmids and primers used in this study are listed in Supplementary Tables S1 and S2, respectively (available with the online version of this paper).

General methods. Preparation of B. subtilis competent cells was carried out by using the two-step method described by Harwood (1990). Antibiotics were supplied by Sigma, and used at the following concentrations: $5 \mu \mathrm{g}$ chloramphenicol ml $\mathrm{ml}^{-1}, 0.5 \mu \mathrm{g}$ erythromycin $\mathrm{ml}^{-1}$ plus $12.5 \mu \mathrm{g}$ lincomycin $\mathrm{ml}^{-1}, 100 \mu \mathrm{g}$ spectinomycin $\mathrm{ml}^{-1}$ and $5 \mu \mathrm{g}$ kanamycin $\mathrm{ml}^{-1}$. For the $\beta$-galactosidase assay in plates, $40 \mu \mathrm{g}$ $\mathrm{X}$-Gal ml ${ }^{-1}$ was used.

In most experiments cells were induced to sporulate by resuspension in StM medium, as described by Sterlini \& Mandelstam (1969). Alternatively, DSM was used (Schaeffer et al., 1965). Spore titre was determined by heat treatment $\left(20 \mathrm{~min}\right.$ at $\left.80^{\circ} \mathrm{C}\right) 18 \mathrm{~h}$ after sporulation initiation.

FapR production and purification. Full-length FapR was produced from plasmid pGES229 as an $\mathrm{N}$-terminal $\mathrm{His}_{6}$-fusion protein in $E$. coli, and it was affinity purified on a Ni-NTA resin, according to the manufacturer's protocol (Qiagen). Protein concentrations were determined by using the Bradford assay (Bradford, 1976), with BSA as a standard.

Western blot analyses, and $\boldsymbol{\beta}$-galactosidase assays. Western blot analyses were performed as previously described (Schujman et al., 2001). GFP antibodies were raised in rabbits that had been injected with GFP, and they were used at a dilution of $1: 1000$. Proteins were detected using alkaline-phosphatase-conjugated antirabbit secondary antibodies (Bio-Rad)

$\beta$-Galactosidase specific activity $\left[\Delta A_{420} \mathrm{~min}^{-1}(\mathrm{ml} \text { culture })^{-1} \times 1000 /\right.$ $\mathrm{OD}_{525}$ ] was determined as described by Miller (1972), after pelleting cell debris.

In vitro transcription. In vitro transcription assays were performed as described by Opdyke et al. (2001). The templates for the reactions were DNA fragments containing the promoter regions of fapR (192 bp) and acpP (255 bp); the fragments were amplified by PCR using primer pairs ylpC116U-ylpC116L and PacpEco-PextACP, respectively (Supplementary Table S2). Purified template DNA was added to the reaction buffer at a final concentration of $13 \mathrm{nM}$ for $\mathrm{PacpP}$, and $4.3 \mathrm{nM}$ for $\mathrm{P} f a p R$, and then incubated in the absence and presence of FapR (213 nM final concentration). Transcription was initiated by adding RNA polymerase purified from B. subtilis, NTPs and $\left[\alpha-{ }^{32}\right.$ P]UTP (Perkin-Elmer), as previously described (Schujman et al., 2006). The RNA transcripts were precipitated, resuspended in formamide loading buffer, and subjected to electrophoresis in a $6 \%$ polyacrylamide gel containing $7 \mathrm{M}$ urea. Radioactive signals were detected by exposing the gel to a PhosphorImager screen, and they were digitalized by using a Storm 840 scanner (GE Healthcare). Quantification of the corresponding signals was carried out using ImageQuant software (version 5.2) (www.imsupport.com).

Growth and metabolic labelling of mutant strains. BMM94 (acpP:: kan amyE:: Pxyl-acpP-cat) was grown overnight on LB agar containing kanamycin and $0.3 \%(\mathrm{w} / \mathrm{v})$ xylose. Cells were resuspended in $\mathrm{LB}$ medium without xylose to an $\mathrm{OD}_{525}$ of 0.05 , and incubated at $37{ }^{\circ} \mathrm{C}$ for $2 \mathrm{~h}$ in the absence of inducer to dilute the pre-existing protein. Then, the culture was divided into two parts, and acpP expression was induced in one half of the culture by the addition of $0.3 \%(\mathrm{w} / \mathrm{v})$ xylose. After incubation for $30 \mathrm{~min}$, cells were incubated with $74 \mathrm{kBq}\left[{ }^{14} \mathrm{C}\right]$ acetate $\mathrm{ml}^{-1}$ for $60 \mathrm{~min}\left(\left[2-{ }^{14} \mathrm{C}\right]\right.$ acetate with a specific activity $2.035 \mathrm{TBq} \mathrm{mol}^{-1}$ was obtained from American Radiolabeled Chemicals). Cells were collected, and the total lipids were extracted by using the Bligh and Dyer method (Bligh \& Dyer, 1959). Radioactivity incorporated into the total lipids was quantified by using a scintillation counter, and the results were expressed as c.p.m. $\left(\mathrm{OD}_{525}\right.$ unit) ${ }^{-1}$. For sporulation studies of strain BMM94 and derivatives, cells were grown in $\mathrm{CH}$ medium (Harwood, 1990), in the absence of xylose, until ACP became limiting for growth. The cells were then harvested, and resuspended in Sterlini-Mandelstam sporulation medium (StM) in the presence or absence of the inducer. Sporulation frequency was measured $24 \mathrm{~h}$ after initiation of sporulation. BMM105 $(\triangle \mathrm{P} a c p P)$ and GS282 were grown overnight in SMM containing the appropriate antibiotic. Cells were inoculated in fresh minimal medium to an $\mathrm{OD}_{525}$ of 0.1 , and grown at $37^{\circ} \mathrm{C}$. At the indicated time, cells were incubated with $37 \mathrm{kBq}\left[{ }^{14} \mathrm{C}\right]$ acetate $\mathrm{ml}^{-1}$ for one generation period. Cells were collected, and total lipids were extracted, as described above.

Temperature-sensitive phenotypes of $B$. subtilis strains bearing mutant forms of ACP were tested at $42{ }^{\circ} \mathrm{C}$, as reported for E. coli (De Lay \& Cronan, 2006), or at higher temperatures, such as 44 or $50{ }^{\circ} \mathrm{C}$, as $\mathrm{B}$. subtilis is able to grow at temperatures of up to $56{ }^{\circ} \mathrm{C}$.

RNA analysis. Total B. subtilis RNA was isolated from cells grown in LB medium, as described by Raya et al. (1998). For Northern blot analyses, $15 \mu \mathrm{g}$ total RNA was separated under denaturing conditions in a $1.2 \%$ agarose gel containing $1.1 \%$ formaldehyde, and transferred to a Hybond-N + membrane (Amersham). RNA blots were hybridized with ${ }^{32} \mathrm{P}$-labelled DNA fragments, using random hexanucleotides as primers for Klenow DNA polymerase. Probe 1 was a 360 bp fragment obtained from the chromosome of strain JH642 by PCR amplification using the oligonucleotides ACPBglIIF and ACPBamHI, which included the coding region of acpP. Probe 2 was a $1000 \mathrm{bp}$ fragment obtained by PCR amplification from the chromosome of strain JH642 using the oligonucleotides FabDUpp and FabDLw, which included part of the coding region of $p l s X$ and fabD (Supplementary Table S2). Hybridization of the membranes was carried out at $65{ }^{\circ} \mathrm{C}$ in a solution containing $5 \times \operatorname{SSPE}[0.9 \mathrm{M} \mathrm{NaCl}$, $0.05 \mathrm{M}$ sodium phosphate (pH 7.7), $5 \mathrm{mM}$ EDTA], $0.5 \% \mathrm{SDS}, 5 \times$ Denhardt's solution, and $20 \mathrm{mg}$ salmon sperm DNA $1^{-1}$. Final washing of the membranes was conducted at $65{ }^{\circ} \mathrm{C}$ in $0.1 \times \mathrm{SSPE}$ 
Table 1. Bacterial strains

\begin{tabular}{|c|c|c|}
\hline Strain & Relevant characteristics & Source or reference \\
\hline \multicolumn{3}{|l|}{ B. subtilis } \\
\hline JH642 & $\operatorname{trpC}$ pheA1 & Laboratory stock \\
\hline GS69 & $\mathrm{P} a c p P-a c p P-g f p-s p c$ & This study \\
\hline GS282 & pMutin:: fabHB-lacZ-ermC & Schujman et al. (2003) \\
\hline GS277 & $a m y E:: \mathrm{P} f a p R-l a c Z-s p c$ & Schujman et al. (2003) \\
\hline GS285 & pMutin:: fabHB-lacZ-ermC fapR:: cat & Schujman et al. (2003) \\
\hline GS367 & $\Delta$ fapR-cat & Schujman et al. (2006) \\
\hline GS380 & pMutin: : fabHB-lacZ-ermC fapR:: cat thrC::Pxyl-fapR7-spc & Schujman et al. (2006) \\
\hline MF56 & $s p o I I G B-g f p-s p c$ & Fujita \& Losick (2002) \\
\hline MF339 & $a m y E::$ Pspac $-g f p-s p c$ & Fujita \& Losick (2002) \\
\hline MO1190 & $\Delta s p o I I G A$ & Guerout-Fleury et al. (1996) \\
\hline BMM18 & thrC: : PacpP-lacZ-erm C & This study \\
\hline BMM44 & $a m y E:: \mathrm{P} a c p P-g f p-s p c$ & This study \\
\hline BMM62 & amyE : : PspoIIQ-acpP-gfp-cat & This study \\
\hline BMM64 & spoIIAC : : kan amyE $:: \mathrm{P} s p a c^{c}-g f p-s p c$ & This study \\
\hline BMM88 & spoIIAC: : kan amyE : : $\mathrm{Pacp} P-g f p-s p c$ & This study \\
\hline BMM93 & amyE:: Pxyl-acpP-cat & This study \\
\hline BMM94 & acpP :: kan amyE : : $\mathrm{P} x y l-a c p P-c a t$ & This study \\
\hline BMM99 & $\Delta \mathrm{P} a c p P-k a n$ & This study \\
\hline BMM101 & $\Delta$ SpoIIGA amyE:: PacpP-gfp-spc & This study \\
\hline BMM102 & $\Delta S p o I I G A$ amyE::Pspac $c^{c}-g f p-s p c$ & This study \\
\hline BMM105 & $\Delta \mathrm{P} a c p P-k a n$ fabHB-lacZ-ermC & This study \\
\hline BMM106 & acpP:: kan amyE::Pxyl-acpP-cat spoIIGB-gfp-spc & This study \\
\hline BMM109 & thrC: : Pxyl-acpP-spc & This study \\
\hline BMM111 & thrC::Pxyl-acpP-spc acpP:: kan & This study \\
\hline BMM115 & $\Delta f a p R$-cat amyE::PfapR-lacZ-spc & This study \\
\hline BMM118 & acpP :: kan thrC::Pxyl-acpP-spc amyE::Pspac hy -acp E. coli & This study \\
\hline BMM119 & acpP:: kan thrC::Pxyl-acpP-spc amyE:: Pspac ${ }^{h y}-$ acp A68T N73D E. coli & This study \\
\hline BMM120 & acpP:: kan thrC::Pxyl-acpP-spc amyE::Pspac ${ }^{h y}-$ acp D38V E. coli & This study \\
\hline BMM122 & acpP:: kan thrC::Pxyl-acpP-spc amyE::Pspac ${ }^{h y}-$ acpP JH642 & This study \\
\hline BMM123 & acpP:: kan thrC::Pxyl-acpP-spc amyE::Pspac ${ }^{h y}-a c p P$ D38V B. subtilis & This study \\
\hline BMM124 & $\Delta \mathrm{P} a c p-k a n$ fabHB-lacZ-ermC amyE::Pxyl-acpP-spc & This study \\
\hline BMM125 & $\Delta \mathrm{P} a c p-k a n$ fabHB-lacZ-ermC thrC: : Pxyl-fapRWT-spc & This study \\
\hline BMM126 & $\Delta \mathrm{Pacp}$-kan fabHB-lacZ-ermC thrC::Pxyl-fapR7-spc & This study \\
\hline BMM127 & $\Delta \mathrm{P} a c p-k a n$ fabHB-lacZ-ermC thrC::Pxyl-fapRWT-spc amyE::Pspac ${ }^{\text {hy }}$-acpP-cat & This study \\
\hline BMM128 & $\Delta \mathrm{Pacp}$-kan fabHB-lacZ-ermC thrC::Pxyl-fapR7-spc amyE::Pspachy-acpP-cat & This study \\
\hline BMM129 & thrC: : Pxyl-acpP-spc ter- $\Delta \mathrm{P} a c p P-k a n$ & This study \\
\hline BMM131 & $\Delta$ fapR:: cat thrC::PacpP-lacZ-ermC & This study \\
\hline RG19148 & spoIIAC: : kan & Arabolaza et al. (2003) \\
\hline \multicolumn{3}{|l|}{ E. coli } \\
\hline MG1655 & Wild-type & Strain collection \\
\hline M15 & Used for expression of fapR & Qiagen \\
\hline NRD28 & $\operatorname{acp} P(\mathrm{ts}) A 68 T$ N73D $\Delta f a b F:: c a t$ & De Lay \& Cronan (2006) \\
\hline NRD29 & $\operatorname{acp} P(\mathrm{ts}) D 38 \mathrm{~V} \Delta \mathrm{fabF}: \mathrm{cat}$ & De Lay \& Cronan (2006) \\
\hline
\end{tabular}

plus $0.1 \%$ SDS. Radioactive signals were detected by exposing the nitrocellulose membranes to a PhosphorImager screen. The size of the acp $P$ transcript was determined by comparison with RNA molecular mass standards (Promega).

Fluorescence microscopy. Sporulation was induced according to the method of Sterlini \& Mandelstam (1969). At appropriate time points during sporulation, $0.5 \mathrm{ml}$ aliquots of the sporulating cultures were removed, centrifuged briefly, and resuspended in $0.05 \mathrm{ml}$ PBS supplemented with the membrane stain FM4-64 $\left(1 \mu \mathrm{g} \mathrm{ml}^{-1}\right.$; Molecular Probes). The concentrated cell suspension was placed on a microscope slide that had been covered with a thin layer of $1 \%$ agarose.

RT-PCR. For RT-PCR analysis, RQ1-DNase (Promega) was added to each RNA preparation at a final concentration of $0.05 \mathrm{U}(\mu \mathrm{g}$ RNA $)^{-1} \mu \mathrm{l}^{-1}$. The preparations were incubated for $30 \mathrm{~min}$ at $37^{\circ} \mathrm{C}$, and then inactivated by adding EGTA to a final concentration of $2 \mathrm{mM}$, and heating the mixture at $65{ }^{\circ} \mathrm{C}$ for $10 \mathrm{~min}$. The amount of extracted RNA was determined by measuring $A_{260}$. RT was performed with $2.4 \mu \mathrm{g} R \mathrm{RN}, 0.5 \mu \mathrm{g} \mathrm{dN}_{6}$ primers, and $8 \mathrm{U} \mathrm{M}-\mathrm{MLV}$ reverse transcriptase (Promega). PCRs on the RT products were 
performed using primer pairs ylpC5Hind and plsXBglII, pplsXUp and fabDmutB, fabGUp and fabGmut, and PacpEco and PacpSph (Supplementary Table S2). A PCR performed using equal amounts of non-retrotranscribed RNA was used as a control for genomic DNA contamination of the RNA samples. To detect the whole operon transcript, primers FapRT137 and PacpSph (Supplementary Table S2) were used, as described in the protocol developed by Aguena \& Spira (2003).

\begin{abstract}
Identification of the transcriptional start site using RNA-ligasemediated rapid amplification of cDNA ends. RNA was extracted from strain JH642, which had been grown to $\mathrm{OD}_{600} 1.5$ at $37^{\circ} \mathrm{C}$ in $15 \mathrm{ml} \mathrm{LB}$ medium, and the acp $P$ transcription start site was mapped using the GeneRacer kit (Invitrogen). All experimental steps were performed according to the manufacturer's instructions. This technique is based on RNA-ligase-mediated and oligocapping rapid amplification of cDNA ends (RACE) methods, and it results in the selective ligation of an RNA oligonucleotide to the $5^{\prime}$ ends of decapped mRNA using T4 RNA ligase. Synthesis of first-strand cDNA was performed with the gene-specific reverse primer ACPGFPEco, and direct amplification of tailed cDNA using a nested gene-specific primer ACPD38VR, and an abridged anchor primer. PCR products were cloned into pCR BluntII TOPO (Invitrogen), and five clones were sequenced. The first nucleotide in the sequence that came after the ligated RNA oligonucleotide sequence was taken as the transcriptional start site.
\end{abstract}

\section{RESULTS}

\section{acpP is transcribed from two independent promoters}

It has been previously reported that $a c p P$, the gene encoding B. subtilis ACP, is contained in a cluster of lipid biosynthesis genes that is located downstream of $p l s X, f a b D$ and $f a b G$ (Morbidoni et al., 1996). In a later study, we found that $f a p R$, the gene encoding the global regulator of fatty acid and phospholipid biosynthesis, is localized upstream in the same cluster (Schujman et al., 2003). Several observations have strongly suggested that $f a p R$, $p l s X, f a b D$ and $f a b G$ are co-transcribed in an operon (previously named $f a p R$ ): the intergenic distance between each of the genes is less than $22 \mathrm{nt}$, and expression of the genes is under the transcriptional control of FapR, responding to the same stimuli (Schujman et al., 2003). Moreover, in a strain containing a deletion of $f a p R$, which has a polar effect on the transcription of downstream genes, expression of $p l s X, f a b D$ and $f a b G$ is diminished (Schujman et al., 2008). In contrast, acpP is separated by $87 \mathrm{nt}$ from the $3^{\prime}$ end of $f a b G$, and a $\sigma^{\mathrm{A}}$-dependent promoter has been proposed to exist in this intergenic sequence (Jarmer et al., 2001; Morbidoni et al., 1996); this suggests that acpP is transcribed independently (Fig. 1a). To address this matter, we performed Northern blot analysis using probes against different genes of the cluster. A probe containing the full sequence of $a c p P$ annealed to an RNA fragment of approximately $300 \mathrm{bp}$, which correlates with the length of acpP mRNA, while no signal was detected for longer RNA fragments (Fig. 1b). Moreover, we did not detect any signal with probes containing the $f a b G$ sequence (data not shown) or the $p l s X$ $f a b D$ region (Fig. 1b). These results suggest that fapR-plsX-
fabDG mRNA is labile, or that it is present in very low amounts, and the results strongly indicate that acpP is transcribed as a single gene, in agreement with previous results (Morbidoni et al., 1996). As described below, confirmation of the existence of an independent promoter for the acpP gene came from the analysis of gene reporter fusions to the $f a b G-a c p P$ intergenic region.

To test whether the cluster of lipid genes is transcribed as an operon, and to find out if acp $P$ is part of the operon, we performed RT-PCR assays. In these experiments, we determined the existence of mRNA containing the intergenic region between each gene of the cluster, and also mRNA comprising the whole cluster (Fig. 1c). Taken together, our results demonstrate that $f a p R, p l s X, f a b D$, $f a b G$ and $a c p P$ comprise the $f a p R$ operon, and that $a c p P$ is transcribed from two different promoters: (1) PfapR, which is shared with the rest of the lipid biosynthesis cluster; and (2) PacpP, which is specific for the gene (Fig. 1a).

\section{PacpP is a FapR-independent strong promoter}

Although acp $P$ is transcribed from $\mathrm{P} f a p R$ and $\mathrm{Pacp} P$, previous results have suggested that acpP expression is not significantly regulated by FapR (Schujman et al., 2003). Those observations seem to indicate that expression from $\mathrm{Pacp} P$ is independent of FapR, and that this promoter is stronger than $\mathrm{P} f a p R$ in physiological conditions. To confirm these hypotheses, we constructed four strains bearing PacpPlac $Z$ or $\mathrm{P} f a p R$-lacZ fusions in a wild-type or a $f a p R^{-}$ background. The strains were grown in LB broth, and assayed for $\beta$-galactosidase activity. There were no appreciable differences in the growth curves of the four strains (data not shown). The pattern of expression from the PacpP promoter in wild-type and $f a p R^{-}$strains was similar, confirming that PacpP expression is not regulated by FapR (Fig. 2a). In contrast, the levels of $\beta$-galactosidase expressed from a PfapR-lacZ fusion were 5- to 10 -fold higher in the $f a p R^{-}$strain than in the wild-type strain (Fig. 2a). These results were confirmed by in vitro transcription assays: transcription from $\mathrm{P} f a p R$ was inhibited in presence of purified FapR, while transcription from PacpP was unaffected by the repressor (Fig. 2b). In wild-type strains, $\beta$ galactosidase specific activity from the $\mathrm{Pacp} P$-lac $Z$ fusion was about 10-fold higher than that from PfapR-lacZ (Fig. 2a). This result agrees with the results obtained with Northern blot experiments, in which a band corresponding to acp $P$ mRNA was readily observed, while no signal was detected for the fapR operon mRNA. Together, these results indicate that while the expression of $f a p R$ operon is coordinated with that of the fap regulon (Schujman et al., 2003), acpP is also expressed independently from the strong $\mathrm{PacpP}$ promoter.

\section{Determination of the transcription start point of acpP}

In order to characterize the $\mathrm{Pacp} P$ promoter, the transcription start point (tsp) of the acpP gene transcript 
(a)

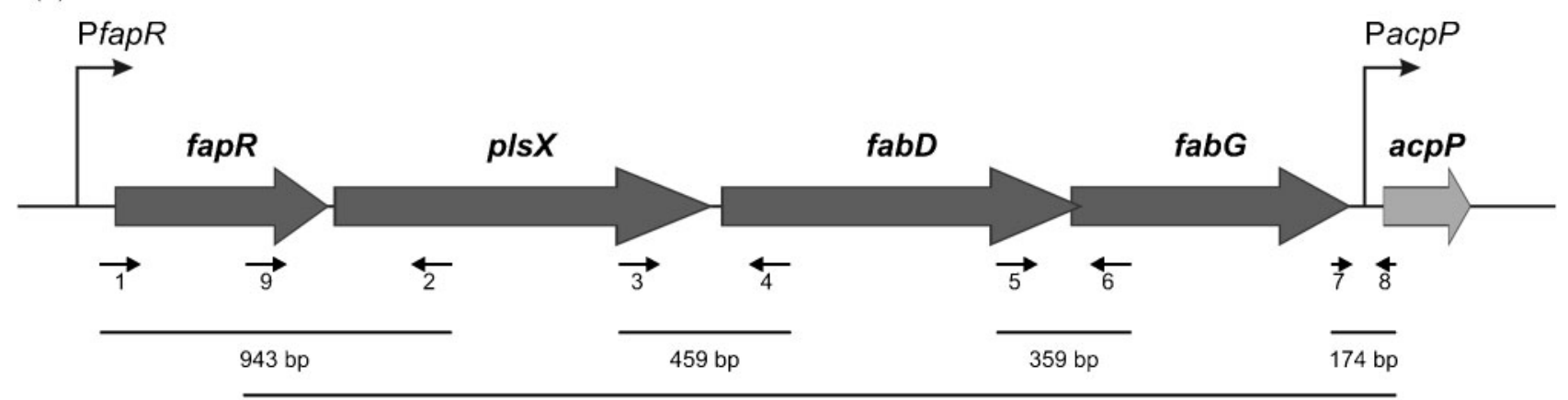

$2991 \mathrm{bp}$

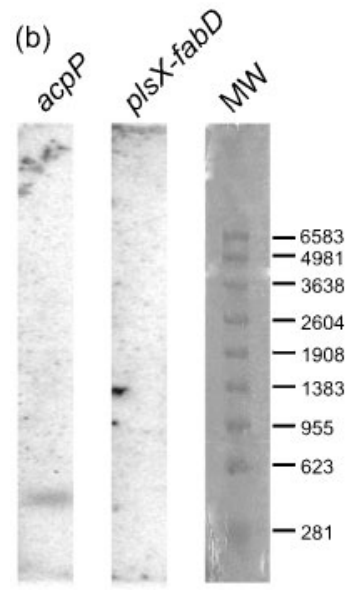

(c)

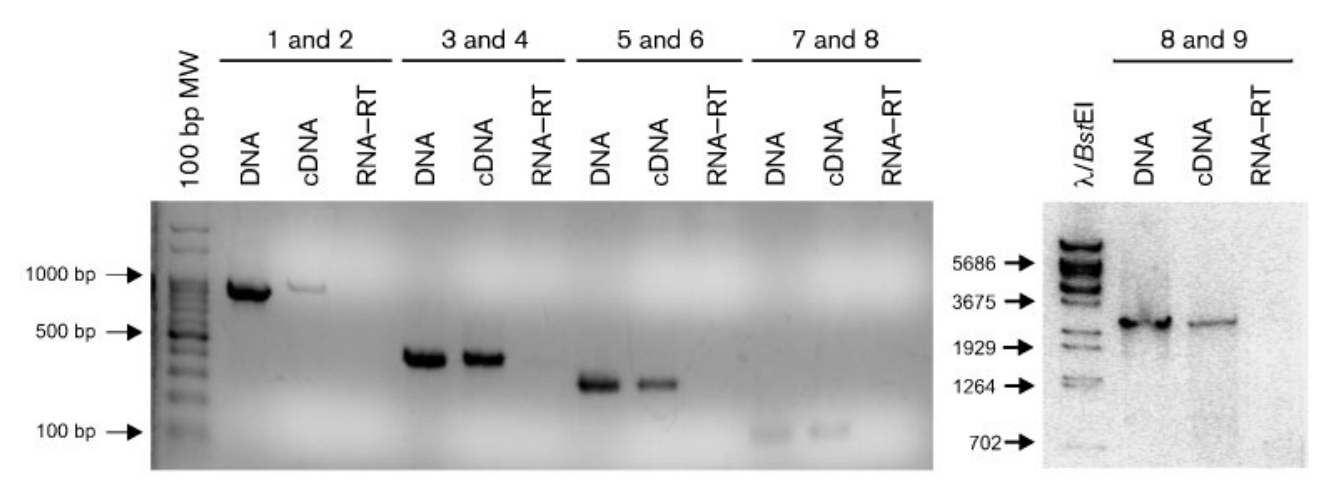

Fig. 1. Genetic organization of the fapR-pls $X$-fabD-fabG-acpP operon. (a) Schematic representation of the fapR operon. Numbered arrows indicate the annealing regions of the oligonucleotide primer pairs utilized for the RT-PCR experiments. The primers were as follows: 1, ylpC5Hind; 2, plsXBglll; 3, pplsXUp; 4, fabDmutB; 5, fabGUp; 6, fabGmut; 7, PacpEco; 8, PacpSph; 9, FapRT137. The predicted sizes of the amplified DNA fragments are indicated. (b) Analysis of the mRNA transcripts derived from the fapR operon. RNA from the exponentially growing wild-type strain $\mathrm{JH} 642$ was isolated, and the full coding sequence of $a c p P$, and a sequence containing the $3^{\prime}$ half of $p / s X$ and the $5^{\prime}$ half of $f a b D$, were used to prepare the ${ }^{32} \mathrm{P}$-labelled probes. Northern blotting was carried out with the individual probes, as described in Methods. Transcripts for $p / s X$ and fabD were below detection limits, and the same result was obtained using RNA from the fap $R^{-}$strain GS367. (c) RT-PCR of fapR operon intergenic sequences. cDNA was synthesized using $\mathrm{dN}_{6}$ primers and RNA of strain $\mathrm{JH} 642$ as the template. PCR was performed with the oligonucleotide pairs 1 and 2, 3 and 4, 5 and 6, and 7 and 8, as shown in (a). cDNA for the RT-PCR of the whole fapR operon was synthesized, as described in Methods. PCR was performed with the oligonucleotides PacpSph and FapRT137 [8 and 9, respectively, in (a)]. DNA from strain JH642 or GS367 was used as a positive control (DNA), and RNA that had not been treated with reverse transcriptase was used as a negative control (RNA-RT).

was determined. We performed five independent $5^{\prime}$ RACE experiments, and all sequences were mapped to the same nucleotide: the adenine nucleotide $22 \mathrm{nt}$ upstream of the translation start (Fig. 3); we propose that this nucleotide is the tsp. Consensus -10 and -35 sequences of the $\sigma^{\mathrm{A}}$ dependent $B$. subtilis promoter are present upstream of the acp $P$ transcriptional start, and a consensus Shine-Dalgarno (SD) sequence is located downstream of the tsp, separated by nine bases from the translation start codon (Fig. 3). In agreement with these results, in vitro transcription assays using purified $B$. subtilis $\sigma^{\mathrm{A}}$-RNA polymerase, and the complete $f a b G-a c p P$ intergenic region as a template, produced mRNA of a length compatible with that expected for the identified tsp (data not shown). The genetic elements described, and operon organization, are not restricted to B. subtilis. Analysis of the genomes of all bacteria belonging to the phylum Firmicutes, and containing a $f a p R$ homologue, indicates that they all share the pentacistronic operon structure, the same $\mathrm{PacpP}$ promoter, and a perfect consensus for the SD sequence (Supplementary Fig. S1).

\section{ACP is essential for growth in B. subtilis}

In $E$. coli, $a c p P$ is an essential gene because its product is the only ACP present in this organism, and it is indispensable 
(a)

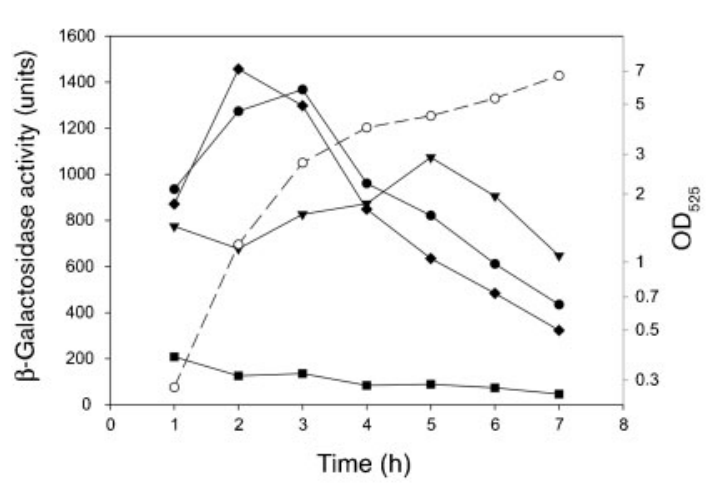

(b)

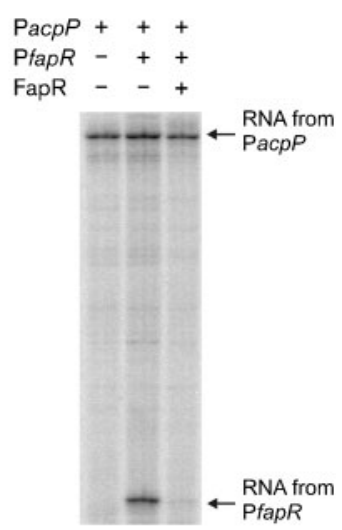

Fig. 2. $P a c p P$ is a strong promoter not regulated by FapR. (a) $B$. subtilis strains $B M M 18\left(f a p R^{+}, \bullet\right)$ and $B M M 131\left(f a p R^{-}, \bullet\right)$ harbouring a PacpP-lacZ fusion, and strains GS277 (fap $\left.R^{+}, \mathbf{\square}\right)$ and BMM115 (fap $\left.R^{-}, \boldsymbol{\nabla}\right)$ containing a PfapR-lacZ fusion, were grown in LB medium at $37{ }^{\circ} \mathrm{C}$. At the indicated times, samples of each culture were collected, and assayed for $\beta$-galactosidase activity (filled symbols). Growth was monitored by measuring $O D_{525}$, and a representative curve is shown $(\bigcirc)$. A single result representative of at least three independent experiments is shown. Replicate values varied by not more than $15 \%$ about the mean. (b) In vitro transcription assays were performed with the fabG-acpP intergenic region or PfapR promoter as the template, in the absence and presence of FapR. The radioactive signal from each transcript, from two independent experiments, was quantified as described in Methods. The ratios of signal intensities of the in vitro transcription performed in the presence versus the absence of FapR were $0.85 \pm 0.05$ for PacpP, and $0.04 \pm 0.01$ for PfapR.

for fatty acid biosynthesis (De Lay \& Cronan, 2006). In the genome of $B$. subtilis, several sequences encode ACP homologues, although most of the homologues are involved in secondary metabolism, such as polyketide and non-ribosomal peptide synthesis (Mootz et al., 2001). To determine if acpP is essential in B. subtilis, we constructed the strain BMM94, in which acpP expression is controlled by the xylose-inducible $\mathrm{P} x y l$ promoter. This strain required xylose for growth, indicating that the acpP gene is essential in B. subtilis (Fig. 4). We confirmed that the growth defect was due to reduction of fatty acid biosynthesis, by labelling cultures of BMM94 with $\left[2-{ }^{14} \mathrm{C}\right]$ acetate, in the presence and absence of xylose. Radioactivity incorporated into lipids of the non-induced cells was less than $10 \%$ of that detected when acpP expression was induced (Fig. 4, inset). In contrast, incorporation of $\left[2-{ }^{14} \mathrm{C}\right]$ acetate into non-lipid (essentially protein) cell components of ACP-depleted cells was above $50 \%$ of that detected in cells expressing acpP (data not shown); this confirmed that the cells were metabolically active, but that they were specifically blocked in lipid biosynthesis. These results indicate that the only acyl carrier protein of B. subtilis that is able to interact with the fatty acid synthase to produce new fatty acids is the product of the acpP gene.

As described above, strain BMM94 allowed us to examine the effects of depletion of the $a c p P$ product on cell growth and lipid synthesis. However, this strategy is not suitable for rapid inactivation of ACP function, as depletion of both mRNA and previously synthesized protein has to take place after elimination of the inducer. Therefore, we tried to construct temperature-sensitive ACP mutants. A recent report has shown that amino acid substitutions $\mathrm{D} 38 \mathrm{~V}$ and A68T,N73D in E. coli ACP result in proteins that confer a temperature-sensitive phenotype (De Lay \& Cronan, 2006). Because the D38 residue is conserved in B. subtilis ACP, we generated strain BMM123, which is a BMM94 derivative that expresses ACP[D38V] under the control of the IPTGinducible Pspac promoter. As expected, strain BMM123 did not grow in the absence of inducers, but when xylose (which induces ACP gene expression) or IPTG (which

\section{$a c p P$

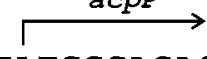 \\ TCGTAGTTTCTCTAGTTTTTAAAAACGAATCCACTATAATACTTGAGGGGAGGTGAATTGCTATGGCAGACA $-35$ $-10$

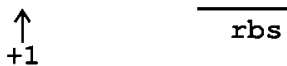

Fig. 3. Determination of the PacpP transcription start site. Sequence of the Pacp $P$ promoter region. The -10 and -35 consensus sequences for $\sigma^{\mathrm{A}}$-RNA polymerase are underlined; rbs, Shine-Dalgarno sequence; +1 , transcription initiation site. 


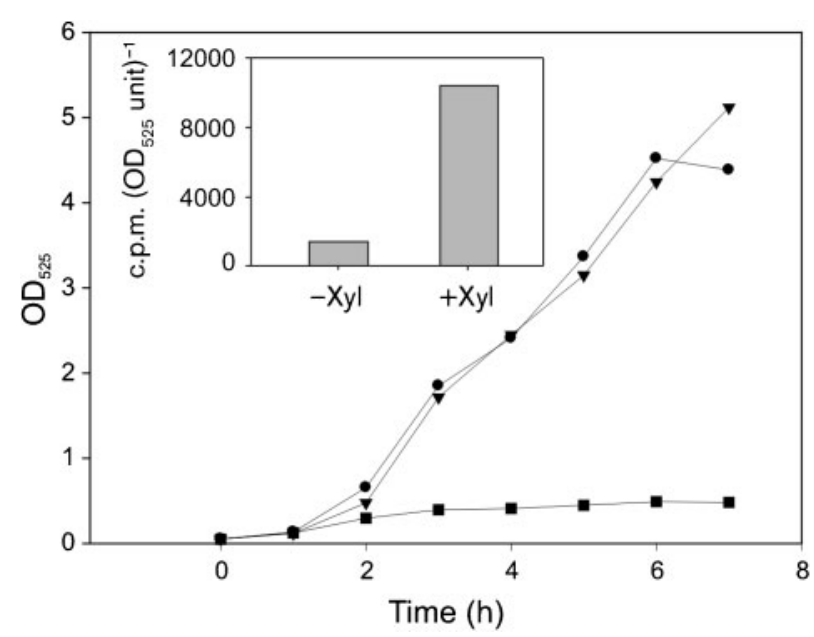

Fig. 4. $a c p P$ is essential in $B$. subtilis. Growth curves in LB medium at $37{ }^{\circ} \mathrm{C}$ of strain $\mathrm{JH} 642$ (wild-type, 0 ), and strain BMM94 (acpP::kan amyE::Pxyl-acpP-cat) in the presence $(\boldsymbol{\nabla})$ and absence ( $\boldsymbol{\square})$ of xylose. Inset: fatty acid biosynthesis is precluded in the absence of ACP. Strain BMM94 was cultured in LB medium in the absence of xylose (Xyl) until ACP became limiting to growth, then xylose $(0.3 \%, w / v)$ was added to half of the culture, and after incubation for $30 \mathrm{~min}$ both cultures were labelled with $\left[2-{ }^{14} \mathrm{C}\right]$ acetate for $30 \mathrm{~min}$. Cells were collected, and radioactivity incorporated into the lipids was measured and normalized, as described in Methods. A single result representative of at least three independent experiments is shown. Replicate values varied by not more than $15 \%$ about the mean.

induces $\mathrm{ACP}[\mathrm{D} 38 \mathrm{~V}]$ gene expression) were added to the culture medium, the bacteria grew normally at $30{ }^{\circ} \mathrm{C}$ (data not shown). However, growth of strain BMM123 in the presence of IPTG was indistinguishable from that of the wild-type strain at 42,44 and $50{ }^{\circ} \mathrm{C}$, indicating that, in contrast to E. coli, the substitution $\mathrm{D} 38 \mathrm{~V}$ in B. subtilis ACP does not result in a temperature-sensitive phenotype. Then, we constructed strains BMM120 and BMM119, which express E. coli ACP[D38V] and ACP[A68T,N73D], respectively, under Pspac control in the BMM94 genetic background. These strains grew normally at $44{ }^{\circ} \mathrm{C}$ when IPTG was the only inducer added (data not shown). These results indicate that although E. coli ACP can fully replace the $B$. subtilis protein in vivo, the thermolability of $E$. coli ACP does not preclude B. subtilis growth. This highlights the different roles of the protein, other than fatty acid biosynthesis, in Gram-negative and Gram-positive bacteria.

\section{PacpP is required for normal lipid homeostasis}

To understand the evolutionary advantage of transcribing acpP from two different promoters, we generated strain BMM105, a GS282 derivative, in which the fabG-acpP intergenic region is deleted (see Methods). We expected that growth of this strain would be severely compromised due to a diminution in the ACP content, but, surprisingly, it grew at the same rate as the wild-type strain (Fig. 5a). Moreover, de novo fatty acid biosynthesis was not reduced in strain BMM105 compared with the wild-type strain (Fig. $5 \mathrm{a}$, inset). These observations indicate that, at least in laboratory conditions, $\mathrm{Pacp} P$ is not necessary for growth or for proper fatty acid biosynthesis. A possible explanation for these results is that $\mathrm{P} f a p R$, in concert with the $f a p$ regulon promoters, is de-repressed in the deletion strain, thus compensating for the lack of acpP expression from PacpP. To test this hypothesis, we constructed strain BMM124, which is a BMM105 derivative containing a Pxyl-acpP fusion at the non-essential amyE locus, and a $\mathrm{P} f a b H B$-lacZ fusion. $\mathrm{fabHB}$ expression, which is under FapR control, was turned off under normal growth conditions, but was greatly increased in a $f a p R^{-}$strain (Fig. 5b); expression of $\mathrm{fabHB}$ is also enhanced when intracellular malonyl-CoA levels increase to induce the release of FapR from its operator sequences (Schujman et al., 2003). In strain BMM124, we found that expression of $\mathrm{P} f a b H B$ was augmented 20 -fold compared with the wildtype strain (Fig. 5b). Inducing the expression of $a c p P$ by addition of xylose to cultures of strain BMM124 reduced $\mathrm{P} f a b H B$ expression to its basal level (Fig. 5b). Based on these results, we propose a model in which the absence of $\mathrm{PacpP}$ reduces acpP expression, slowing down fatty acid biosynthesis, and causing malonyl-CoA accumulation; this is promptly compensated by overexpression of the fap regulon, and this, in turn, increases ACP levels, to reach a new steady state in which fatty acid biosynthesis proceeds at a normal rate, but malonyl-CoA levels and fap regulon expression are increased. To further challenge this model, we used the fapR7 allele, which encodes the superrepressor FapR[G107V,L128W], in which the FapR-malonyl-CoA interaction is disrupted (Schujman et al., 2006). Expression of the fapR7 allele in multicopy plasmids results in a lethal phenotype in B. subtilis cells because of transcriptional inhibition of the fap essential genes (Schujman et al., 2006). However, although expression of fapR7 ectopically integrated into the chromosome of wildtype cells decreased the transcription of the fap regulon (data not shown), the levels of enzymes required for lipid synthesis were sufficient to support normal growth (Fig. 5c, strain GS380). In contrast, the growth of $\triangle \mathrm{P} a c p P$ cells expressing a chromosomal copy of the fapR7 allele (strain BMM126) was impaired (Fig. 5c), suggesting that this strain is unable to produce sufficient ACP to sustain fatty acid biosynthesis. To confirm this, the acpP gene, under the control of the IPTGinducible hyperspac promoter, was integrated ectopically into the amyE locus of strain BMM126 to generate strain BMM128. IPTG-induced production of ACP relieved the deleterious effect of fapR7 on $\triangle \mathrm{P} a c p P$ cells, while, in the absence of IPTG, growth of these cells was severely affected (Fig. 5c). These data demonstrate that malonyl-CoAmediated overexpression of acp $P$ is essential to overcome the defect in lipid synthesis generated by the absence of the $\mathrm{Pacp} P$-specific promoter. Altogether, our results indicate that, although $\mathrm{P} a c p P$ is not essential for $B$. subtilis growth, it contributes to maintain lipid homeostasis in this organism. 
(a)
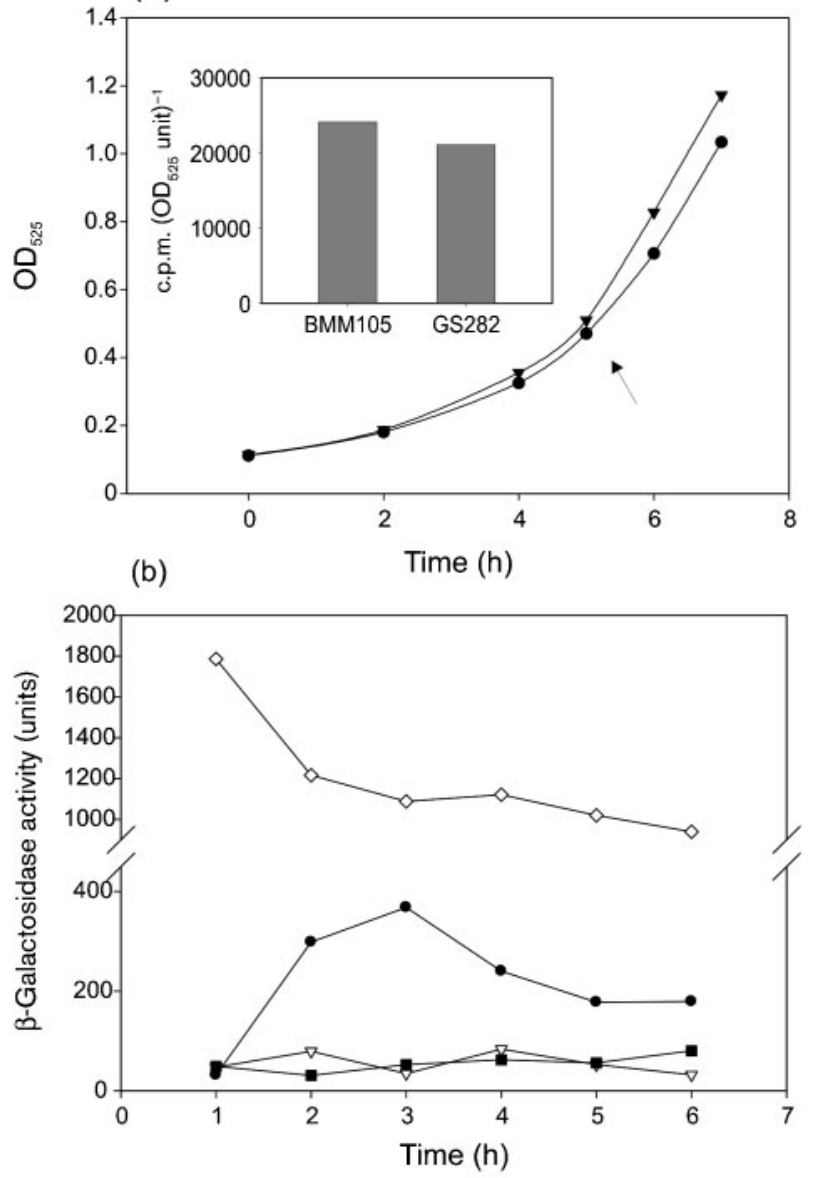

(c)
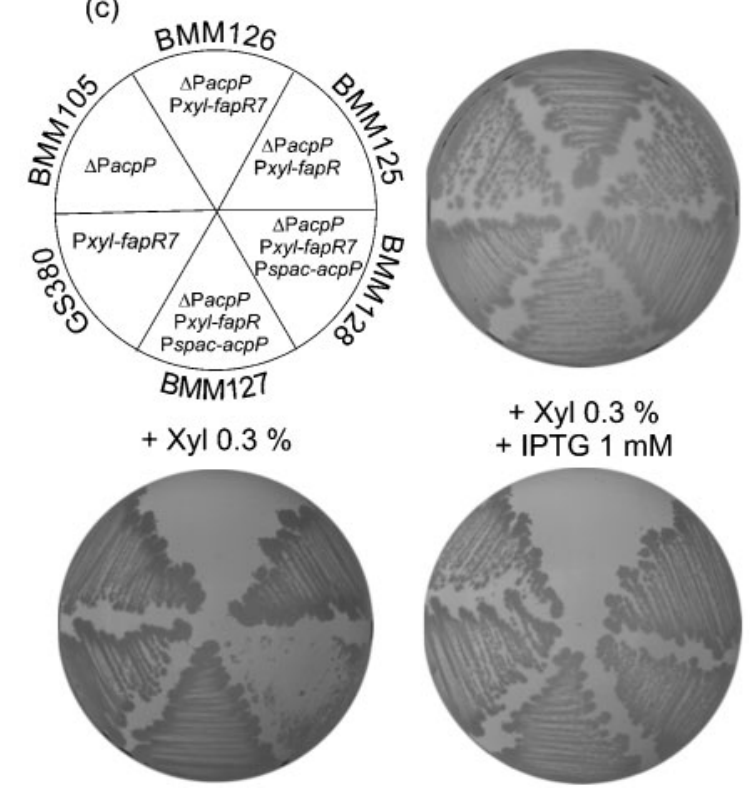

\section{ACP is required for, and is compartmentalized during $B$. subtilis differentiation}

Under nutrient limitation and high cell density, B. subtilis initiates a differentiation process that is termed sporula-
Fig. 5. $P a c p P$ deletion alters fatty acid biosynthesis regulation. (a) B. subtilis strains BMM105 $(\triangle \mathrm{Pacp} P, \mathbf{0})$ and GS282 (wild-type $\mathrm{Pacp} P, \boldsymbol{\nabla})$ were grown in $\mathrm{SMM}$ at $37{ }^{\circ} \mathrm{C}$. Growth was monitored by measuring $\mathrm{OD}_{525}$. $\left[2-{ }^{14} \mathrm{C}\right]$ Acetate was added at the indicated time (arrow). After one generation, cultures were collected, and radioactivity incorporated into the lipids was determined, as described in Methods (see inset). (b) B. subtilis strains GS282 (wild-type), GS285 ( $\triangle f a p R)$ and BMM124 ( $\triangle \mathrm{Pacp} P$ ), harbouring a $\mathrm{PfabHB}-\mathrm{lacZ}$ fusion at the amyE locus, were grown in LB medium, with and without xylose $(0.3 \%, w / v)$ at $37{ }^{\circ} \mathrm{C}$. At the indicated times, samples of each culture were collected, and assayed for $\beta$ galactosidase activity. $\boldsymbol{\square}$, GS282; $\diamond$, GS285; •, BMM124 cultured without xylose; $\nabla, \mathrm{BMM} 124$ cultured with xylose. In (a) and (b), a single result representative of at least three independent experiments is shown. Replicate values varied by not more than $15 \%$ about the mean. (c) Derivatives of strain BMM105 ( $\triangle \mathrm{Pacp} P)$ containing wild-type or superrepressor (fapR7) alleles of fapR under the control of the xylose-inducible Pxy/ promoter, and a copy of the acpP gene fused to the IPTG-inducible Pspac promoter were generated. Strains were grown on LB agar containing no inducers (top-right plate); LB agar plus $0.3 \%(\mathrm{w} / \mathrm{v})$ xylose (Xyl) (bottom-left plate), and LB agar plus $0.3 \%(\mathrm{w} / \mathrm{v})$ xylose and $1 \mathrm{mM}$ IPTG (bottom-right plate), for $24 \mathrm{~h}$ at $37^{\circ} \mathrm{C}$. The names and genotypes of each strain are indicated in the top-left diagram.

tion. This process is characterized by an asymmetrical division in which two compartments are created. The larger compartment, called the mother cell, engulfs and nurtures the smaller compartment, which is known as the forespore, and eventually lyses to release a dormant environmentally resistant spore. In a previous work, we reported that inhibition of FabF (the condensing enzyme involved in fatty acid elongation) impairs sporulation, thus preventing activation of $\sigma^{\mathrm{E}}$, which directs mother-cellspecific gene transcription. The factor $\sigma^{\mathrm{E}}$ is synthesized as a pre-protein, and is cleaved by the membrane protease SpoIIGA in the mother cell only (Schujman et al., 1998). To test if ACP is also necessary for B. subtilis differentiation, we compared the sporulation frequencies of strain BMM94 induced to sporulate in the absence and presence of xylose. We found that in the absence of ACP, sporulation efficiency was less than $0.005 \%$ of that of the corresponding strain induced to sporulate in the presence of the inducer, as judged by the production of heat-resistant c.f.u. (spores). As shown in Fig. 6(a), ACP-depleted cells produced pro- $\sigma^{\mathrm{E}}$ in normal amounts, but they did not process it; this confirmed that de novo fatty acid biosynthesis was essential for $\sigma^{\mathrm{E}}$-directed gene expression.

Previous experiments that have assessed the expression of FASII genes during growth have shown that their transcription is maximal during mid-exponential growth, and is almost turned off after the cells reach stationary phase, and begin to sporulate (Schujman et al., 2001). However, we found a different expression pattern of PacpP in cells growing in LB medium, where the highest transcriptional activity was observed at the end of exponential growth (Fig. 2a). To analyse acpP expression 
(a)

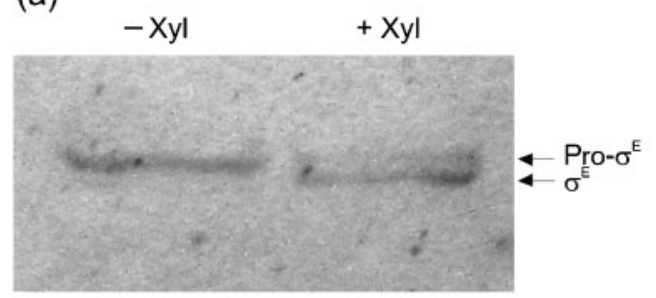

(b)

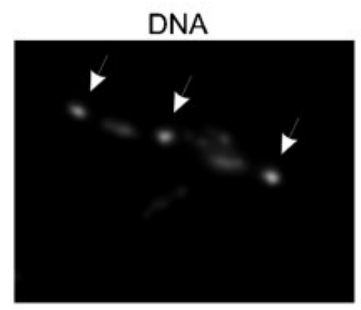

PacpP-acpP-gfp

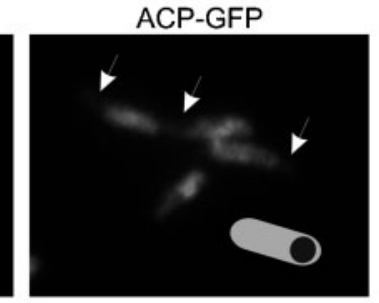

(c)

PspollQ-acpP-gfp

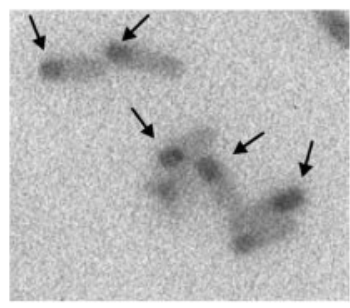

(d)
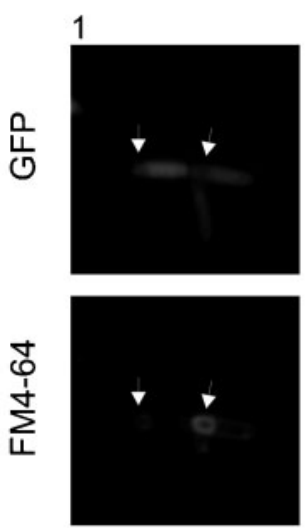

(e)
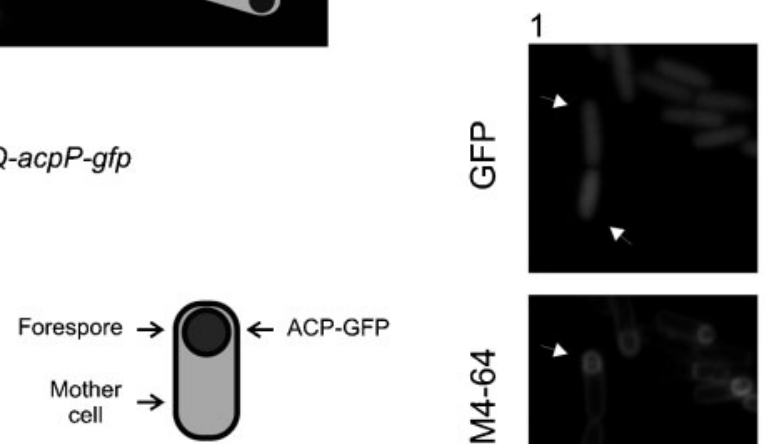

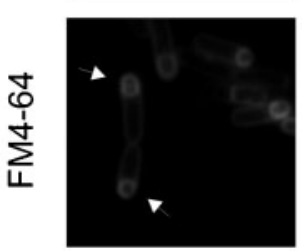

\section{PacpP-gfp}
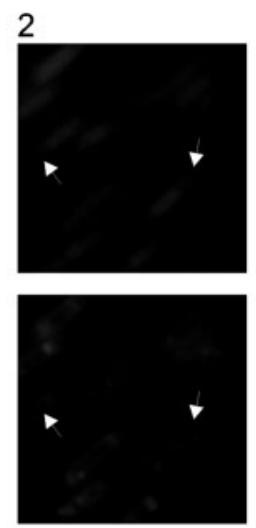

Pspac ${ }^{c}-g f p$
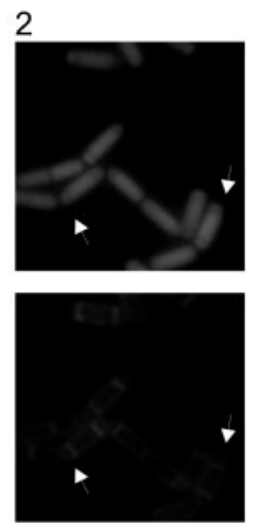

3
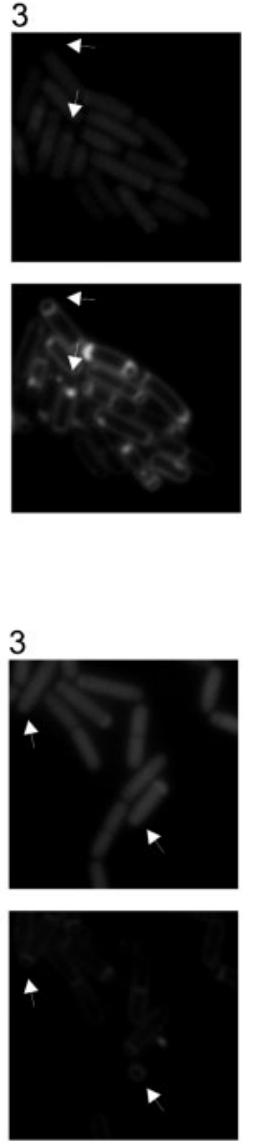

Fig. 6. ACP is necessary for sporulation, and it localizes in the mother cell. (a) Strain BMM94 was induced to sporulate, as described in Methods, and harvested $6 \mathrm{~h}$ after initiation of sporulation. Pro- $\sigma^{\mathrm{E}}$ and $\sigma^{\mathrm{E}}$ content was determined by immunoblotting, as described in Methods. Similar results were obtained for the untreated culture when samples were collected $18 \mathrm{~h}$ after resuspension in sporulation medium. (b) Subcellular localization of ACP-GFP during sporulation. Strain GS69, carrying the PacpP-acpP-gfp fusion, was induced to sporulate by resuspension in StM, and cells were examined $2 \mathrm{~h}$ later by using fluorescence microscopy. The sporangia were treated with 4,6-diamidino-2-phenylindole (DAPI) dye to visualize DNA. Left, fluorescence from DAPI; right, fluorescence from ACP-GFP. The arrows indicate the forespore (condensed DNA in the DAPI image). (c) Effect of the $\sigma^{\mathrm{F}}$-dependent Pspol/Q promoter on the localization of ACP-GFP in strain BMM62 (amyE: : Pspol/Q-acpP-gfp). Cells were induced to sporulate by resuspension in StM, and examined $3 \mathrm{~h}$ later. (d) Distribution of GFP (top panels) generated from the PacpP promoter. Two hours after induction of sporulation by resuspension in StM, the sporangia were treated with the vital membrane stain FM4-64 to visualize membranes (bottom panels). Columns: 1, BMM44 (amyE::PacpP-gfp); 2, BMM88 (sigF spollAC::kan amyE::PacpP-gfp); 3, BMM101 (unable to process $\sigma^{\mathrm{E}} ; \Delta s p o l / G A$ amyE::PacpP-gfp). (e) Distribution of GFP generated from the constitutive $\sigma^{\mathrm{A}}$-dependent Pspac ${ }^{C}$ promoter. Columns:

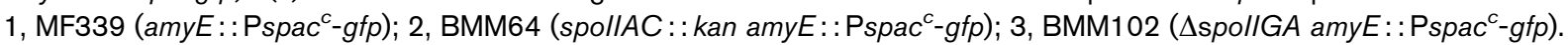
Cells were treated as described in $(d)$.

during sporulation, we turned to DSM medium, in which B. subtilis starts to sporulate at the beginning of stationary phase, after nutrients have been exhausted. In this condition, acp $P$ transcription was maximal at the end of exponential growth, and continued for at least $3 \mathrm{~h}$ after the initiation of sporulation (data not shown). The reporterfusion results were confirmed by fluorescence microscopy experiments, which analysed the signal of the GFP fused to the carboxyl end of ACP, or to the carboxyl end of FabF, which was used as a control. Both tagged proteins were evenly distributed in the cytoplasm during the exponential phase of growth. However, while the signal for FabF-GFP was below the level of detection after sporulation initiation (data not shown), ACP-GFP was readily observed in sporulating cells (Fig. 6b). Strikingly, the distribution of the signal of ACP-GFP in septated cells was not uniform, and it was only barely detectable in the smaller compartment (Fig. 6b). To determine whether this localization pattern 
was due to a differential expression of acpP in both of the compartments, or to the degradation of the ACP-GFP chimera in the forespore, we utilized two different strategies. First, we fused the gene encoding ACP-GFP to a promoter (PspoIIQ) that is recognized by the foresporespecific transcription factor $\sigma^{\mathrm{F}}$ immediately upon septation. By using this fusion, we determined that fluorescence was localized in the forespore (Fig. 6c). This result indicates that, when targeted to the forespore, ACP-GFP is stable, and accumulates in the small chamber of the sporangium. Second, we analysed the localization of GFP when expressed directly from the $\mathrm{Pacp} P$ promoter. The fluorescent probe was found predominantly in the larger compartment (Fig. 6d). Together, these results strongly suggest that localization of the ACP-GFP chimera in the mother cell is due to transcriptional events, rather than to translational or post-translational events.

Next, we sought to examine whether a negative regulator synthesized specifically in the forespore was repressing PacpP, or whether a transcriptional activator of this promoter was specifically acting in the mother cell. To address these questions, we analysed $\mathrm{Pacp} P$ - $g f p$ expression in mutants blocked in cell-specific activation of $\sigma^{\mathrm{F}}$ (which is responsible of forespore-specific gene expression) or $\sigma^{\mathrm{E}}$ (which directs mother-cell-specific gene transcription). As shown in Fig. 6(d), expression from $\mathrm{P} a c p P$ did not change in the mutant cells, suggesting that transcription from $\mathrm{Pacp} P$ is not regulated by a compartment-specific regulator during sporulation. When GFP was transcribed from the $\sigma^{\mathrm{A}}$-dependent constitutive promoter Pspac ${ }^{c}$, we observed only a slight decrease in the fluorescent signal from the forespore after septum formation (Fig. 6e). This suggested that preferential expression of ACP in the mother cell is not common to all $\sigma^{\mathrm{A}}$-dependent promoters, but that it especially increased for its native promoter, $\mathrm{PacpP}$.

\section{DISCUSSION}

In this work, we provide evidence to demonstrate that $a c p P$ is part of the pentacistronic operon fapR-plsX-fabD-fabGacpP. Although mRNA of the expected size for the entire operon was not detected by Northern blots, we demonstrated its existence by RT-PCR (Fig. 1c). We also provide evidence that each gene of the cluster is transcribed concatenated with the adjacent gene (Fig. 1c), and expression of the four initial genes is controlled by the same transcriptional regulator FapR, which interacts with only one operator located at the promoter of the operon (Schujman et al., 2003). Finally, when the intergenic region fabG-acpP was deleted, expression of acpP became regulated by FapR, indicating that it is transcribed exclusively from $\mathrm{P} f a p R$, together with the other four genes of the operon (Fig. 5). It should be noted that de Hoon et al. (2005) predicted, by using bioinformatics, the existence of a transcriptional terminator in the fabG-acpP intergenic region. To discard this hypothesis, we constructed a $\triangle \mathrm{P} a c p P B$. subtilis strain that conserves the putative transcriptional terminator (BMM129, see Methods), and determined that acpP is efficiently transcribed from PfapR (data not shown), demonstrating that the predicted sequence does not encode a functional transcriptional terminator.

Several sequences similar to that of the $a c p P$ gene are found in the genome of B. subtilis (Calderone et al., 2006; Mootz et al., 2001). Most of the sequences encode carrier protein domains that are present in the context of larger proteins, but others, such as $a c p K$, encode free-standing ACPs that are able to receive from malonyl-CoA a malonate group that is processed by other enzymes. These ACP homologues are key components in the production of secondary metabolites, such as polyketides and non-ribosomal peptides (Calderone et al., 2006; Mootz et al., 2001). Here, we showed that, in the absence of acp $P$ expression, growth was inhibited as a consequence of lack of de novo fatty acid biosynthesis (Fig. 4). These experiments demonstrate that acpP is an essential gene in B. subtilis, and confirm that its homologues, such as $a c p K$, are not involved in primary metabolism. De Lay \& Cronan (2006) have shown that ACP is essential in E. coli by the use of thermosensitive versions of the protein. Intriguingly, $B$. subtilis strains containing the same construction as the sole functional ACP were able to grow normally, even at higher temperatures. It is possible that a key enzyme in fatty acid biosynthesis in $E$. coli is unable to interact with $\mathrm{ACP}[\mathrm{D} 38 \mathrm{~V}]$ and $\mathrm{ACP}[\mathrm{A} 68 \mathrm{~T}, \mathrm{~N} 73 \mathrm{D}]$ at $42{ }^{\circ} \mathrm{C}$, but that its counterpart in $B$. subtilis recognizes the mutant proteins at non-permissive temperatures. A second possibility is that the intracellular milieu of $B$. subtilis differs from that of $E$. coli. For example, differences in chaperone proteins, and in protein degradation rates, can suppress temperaturesensitive mutants (Van Dyk et al., 1989). A third plausible explanation is that residual activity of the mutant version of ACP is sufficient for fatty acid biosynthesis in B. subtilis, but not in E. coli. Another possible explanation is that the growth inhibition in E. coli temperature-sensitive mutants at high temperature is, in part, due to an essential function of ACP that is unrelated to lipid biosynthesis, and is not necessary in B. subtilis. For example, ACP is needed for the transfer of acyl intermediates in lipid A synthesis, which is essential for $E$. coli growth, but is not synthesized in Grampositive bacteria. Although ACP is clearly essential in both Gram-negative and Gram-positive bacteria, and the proteins from the different organisms are readily interchangeable (De Lay \& Cronan, 2007), our results imply that the physiology and roles of ACP are not entirely conserved in different bacteria. In agreement with this hypothesis, recent work has shown that, while E. coli has evolved a specific stringent response involving ACP/SpoT interaction, ACP does not interact with the SpoT homologue in B. subtilis (Battesti \& Bouveret, 2008).

From the few micro-organisms for which transcription of the ACP-encoding gene has been analysed (Lu \& Rock, 2006; Rawlings \& Cronan, 1992), a pattern has emerged, in which acpP is contained in a cluster of lipid biosynthesis 
genes, and is expressed from two or more promoters, one of which is strong and exclusive for this gene. Physical association of genes related to the same metabolic route is a common theme in bacteria, and is an advantage for regulating the synthesis of enzymes that act sequentially. Strong promoters are usually linked to the expression of abundant proteins, such as those involved in ribosome synthesis, energy metabolism and transport. In agreement with this, E. coli ACP is one of the most abundant proteins in E. coli, and constitutes about $0.25 \%$ of the total soluble protein (Cronan \& Rock, 1996). Although growth of the $\triangle \mathrm{P} a c p P$ strain was not affected in the conditions tested, we showed in vivo that leaving the acpP gene under the sole control of the FapR-regulated $\mathrm{P} f a p R$ promoter results in overexpression of $\mathrm{fabHB}$ (Fig. 5b), which is co-ordinated with the rest of the fap regulon (Schujman et al., 2003). This compensatory mechanism to increase acp $P$ expression has the adverse consequence of forcing overexpression of the entire fap regulon, and this could be energetically expensive. These results highlight the importance of a dedicated promoter for the $a c p P$ gene, and constitute a new example that emphasizes the role of FapR in bacterial lipid homeostasis.

High intracellular amounts of ACP might be needed because of its role in fatty acid biosynthesis, as the protein has to deliver the acyl intermediates to several different soluble enzymes. Also, ACP interacts with many other proteins that are unrelated to fatty acid biosynthesis (Gully et al., 2003), and it transfers acyl intermediates to other proteins and building blocks (Byers \& Gong, 2007). We demonstrate here that ACP plays a crucial role in the differentiation of B. subtilis. It is not clear yet whether its activity is necessary for fatty acid biosynthesis only, as we have previously demonstrated that this biosynthesis pathway is necessary for sporulation (Schujman et al., 1998), or whether ACP also delivers an acyl intermediate to a key sporulation enzyme to modulate its function. Pro- $\sigma^{\mathrm{E}}$ is present in both of the compartments after asymmetrical septum formation, but it is cleaved by SpoIIGA protease specifically in the mother cell. The mechanism responsible for this compartment-specific activation of $\sigma^{\mathrm{E}}$ remains elusive. We present evidence that ACP is needed for $\sigma^{\mathrm{E}}$ processing (Fig. 6a), and that ACP localizes preferentially in the mother cell (Fig. 6b). Thus, it is tempting to speculate that an acyl-ACP participates in the activation of the $\sigma^{\mathrm{E}}$ cleavage machinery, and restrains its activity to the larger compartment.

The basis for compartmentalization of ACP during sporulation is unclear. It has been reported recently that during passage through the asymmetrical septum, forespore DNA becomes depleted of its associated proteins, including $\sigma^{\mathrm{A}}$. This would diminish $\sigma^{\mathrm{A}}$-dependent transcription in the small compartment (Marquis et al., 2008). Fujita \& Losick (2002), and our own results (Fig. 6e) indicate that this is not the case for the constitutive $\sigma^{\mathrm{A}}$ dependent Pspac promoter. It is possible that the differential expression of $\sigma^{\mathrm{A}}$-dependent promoters in each compartment depends on the strength of the promoter, and is noticeable for only the strong promoters, such as $\mathrm{PacpP}$. Other $\sigma^{\mathrm{A}}$-dependent promoters that are known to be active during sporulation for a longer period in the mother cell than in the forespore are the Spo0A-dependent promoters (Fujita \& Losick, 2003). Spo0A is the master regulator for entry into sporulation, and activates the expression of more than 100 sporulation genes. $\mathrm{PacpP}$ is not known to be controlled by Spo0A (Molle et al., 2003), and in a Spo0A mutant strain, we found that expression from $\mathrm{Pacp} P$ was slightly induced rather than repressed, indicating that Spo0A does not activate its transcription (data not shown). It is worth noting that we tried to purify a $B$. subtilis protein able to bind to the $\mathrm{Pacp} P$ region, but our initial attempts were unsuccessful (data not shown). Identification of the mechanisms that influence transcription of this essential protein in B. subtilis and related bacteria should be of central importance to increase our knowledge of membrane lipid homeostasis, and the role of lipids in sporulation.

\section{ACKNOWLEDGEMENTS}

We gratefully acknowledge Masaya Fujita, from the laboratory of R. Losick, and Nicholas De Lay, from the laboratory of J. Cronan, for kindly providing bacterial strains. This work was supported by Consejo Nacional de Investigaciones Científicas y Técnicas (CONICET, Argentina) and Agencia de Promoción Científica y Tecnológica (FONCYT, Argentina). M.A.M. is a fellow from CONICET, and G. E. S. and D. de M. are Career Investigators at the same institution. D. de $\mathrm{M}$ is an International Research Scholar of the Howard Hughes Medical Institute.

\section{REFERENCES}

Aguena, M. \& Spira, B. (2003). RT-PCR of long prokaryotic operon transcripts without DNase treatment. J Microbiol Methods 55, 419423.

Arabolaza, A. L., Nakamura, A., Pedrido, M. E., Martelotto, L., Orsaria, L. \& Grau, R. R. (2003). Characterization of a novel inhibitory feedback of the anti-anti-sigma SpoIIAA on Spo0A activation during development in Bacillus subtilis. Mol Microbiol 47, 1251-1263.

Battesti, A. \& Bouveret, E. (2008). Bacteria possessing two RelA/ SpoT-like proteins have evolved a specific stringent response involving the acyl carrier protein-SpoT interaction. J Bacteriol 191, 616-624.

Bligh, E. G. \& Dyer, W. J. (1959). A rapid method of total lipid extraction and purification. Can J Biochem Physiol 37, 911-917.

Bradford, M. M. (1976). A rapid and sensitive method for the quantitation of microgram quantities of protein utilizing the principle of protein-dye binding. Anal Biochem 72, 248-254.

Byers, D. M. \& Gong, H. (2007). Acyl carrier protein: structurefunction relationships in a conserved multifunctional protein family. Biochem Cell Biol 85, 649-662.

Calderone, C. T., Kowtoniuk, W. E., Kelleher, N. L., Walsh, C. T. \& Dorrestein, P. C. (2006). Convergence of isoprene and polyketide biosynthetic machinery: isoprenyl-S-carrier proteins in the $p k s X$ pathway of Bacillus subtilis. Proc Natl Acad Sci U S A 103, 89778982 . 
Cronan, J. E. \& Rock, C. O. (1996). Biosynthesis of membrane lipids. In Escherichia coli and Salmonella: Cellular and Molecular Biology, 2nd edn, pp. 612-636. Edited by F. C. Neidhardt and others. Washington, DC: American Society for Microbiology.

de Hoon, M. J., Makita, Y., Nakai, K. \& Miyano, S. (2005). Prediction of transcriptional terminators in Bacillus subtilis and related species. PLOS Comput Biol 1, e25.

De Lay, N. R. \& Cronan, J. E. (2006). Gene-specific random mutagenesis of Escherichia coli in vivo: isolation of temperaturesensitive mutations in the acyl carrier protein of fatty acid synthesis. J Bacteriol 188, 287-296.

De Lay, N. R. \& Cronan, J. E. (2007). In vivo functional analyses of the type II acyl carrier proteins of fatty acid biosynthesis. J Biol Chem 282, 20319-20328.

de Mendoza, D., Schujman, G. E. \& Aguilar, P. S. (2002). Biosynthesis and function of membrane lipids. In Bacillus Subtilis and its Closest Relatives: From Genes to Cells, pp. 43-55. Edited by A. L. Sonenshein, J. A. Hoch \& R. Losick. Washington, DC: American Society for Microbiology.

Fujita, M. \& Losick, R. (2002). An investigation into the compartmentalization of the sporulation transcription factor sigmaE in Bacillus subtilis. Mol Microbiol 43, 27-38.

Fujita, M. \& Losick, R. (2003). The master regulator for entry into sporulation in Bacillus subtilis becomes a cell-specific transcription factor after asymmetric division. Genes Dev 17, 1166-1174.

Guerout-Fleury, A. M., Frandsen, N. \& Stragier, P. (1996). Plasmids for ectopic integration in Bacillus subtilis. Gene 180, 57-61.

Gully, D., Moinier, D., Loiseau, L. \& Bouveret, E. (2003). New partners of acyl carrier protein detected in Escherichia coli by tandem affinity purification. FEBS Lett 548, 90-96.

Harwood, C. R. \& Cutting, S. M. (editors) (1990). Molecular Biology Methods for Bacillus. Chichester, UK: Wiley.

Jarmer, H., Larsen, T. S., Krogh, A., Saxild, H. H., Brunak, S. \& Knudsen, S. (2001). Sigma A recognition sites in the Bacillus subtilis genome. Microbiology 147, 2417-2424.

Lu, Y. J. \& Rock, C. O. (2006). Transcriptional regulation of fatty acid biosynthesis in Streptococcus pneumoniae. Mol Microbiol 59, 551-566.

Marquis, K. A., Burton, B. M., Nollmann, M., Ptacin, J. L., Bustamante, C., Ben Yehuda, S. \& Rudner, D. Z. (2008). SpoIIIE strips proteins off the DNA during chromosome translocation. Genes Dev 22, 1786-1795.

Marrakchi, H., Choi, K. H. \& Rock, C. O. (2002). A new mechanism for anaerobic unsaturated fatty acid formation in Streptococcus pneumoniae. J Biol Chem 277, 44809-44816.

Miller, J. H. (1972). Experiments in Molecular Genetics. Cold Spring Harbor, NY: Cold Spring Harbor Laboratory.

Molle, V., Fujita, M., Jensen, S. T., Eichenberger, P., GonzalezPastor, J. E., Liu, J. S. \& Losick, R. (2003). The Spo0A regulon of Bacillus subtilis. Mol Microbiol 50, 1683-1701.
Mootz, H. D., Finking, R. \& Marahiel, M. A. (2001). 4'-Phosphopantetheine transfer in primary and secondary metabolism of Bacillus subtilis. J Biol Chem 276, 37289-37298.

Morbidoni, H. R., de Mendoza, D. \& Cronan, J. E., Jr (1996). Bacillus subtilis acyl carrier protein is encoded in a cluster of lipid biosynthesis genes. J Bacteriol 178, 4794-4800.

Opdyke, J. A., Scott, J. R. \& Moran, C. P., Jr (2001). A secondary RNA polymerase sigma factor from Streptococcus pyogenes. Mol Microbiol 42, 495-502.

Rawlings, M. \& Cronan, J. E., Jr (1992). The gene encoding Escherichia coli acyl carrier protein lies within a cluster of fatty acid biosynthetic genes. J Biol Chem 267, 5751-5754.

Raya, R., Bardowski, J., Andersen, P. S., Ehrlich, S. D. \& Chopin, A. (1998). Multiple transcriptional control of the Lactococcus lactis trp operon. J Bacteriol 180, 3174-3180.

Schaeffer, P., Millet, J. \& Aubert, J. P. (1965). Catabolic repression of bacterial sporulation. Proc Natl Acad Sci U S A 54, 704-711.

Schujman, G. E., Grau, R., Gramajo, H. C., Ornella, L. \& de Mendoza, D. (1998). De novo fatty acid synthesis is required for establishment of cell type-specific gene transcription during sporulation in Bacillus subtilis. Mol Microbiol 29, 1215-1224.

Schujman, G. E., Choi, K. H., Altabe, S., Rock, C. O. \& de Mendoza, D. (2001). Response of Bacillus subtilis to cerulenin and acquisition of resistance. J Bacteriol 183, 3032-3040.

Schujman, G. E., Paoletti, L., Grossman, A. D. \& de Mendoza, D. (2003). FapR, a bacterial transcription factor involved in global regulation of membrane lipid biosynthesis. Dev Cell 4, 663-672.

Schujman, G. E., Guerin, M., Buschiazzo, A., Schaeffer, F., Llarrull, L. I., Reh, G., Vila, A. J., Alzari, P. M. \& de Mendoza, D. (2006). Structural basis of lipid biosynthesis regulation in Gram-positive bacteria. EMBO J 25, 4074-4083.

Schujman, G. E., Altabe, S. \& de Mendoza, D. (2008). A malonylCoA-dependent switch in the bacterial response to a dysfunction of lipid metabolism. Mol Microbiol 68, 987-996.

Spizizen, J. (1958). Transformation of biochemically deficient strains of Bacillus subtilis by deoxyribonucleate. Proc Natl Acad Sci U S A 44, 1072-1078.

Sterlini, J. M. \& Mandelstam, J. (1969). Commitment to sporulation in Bacillus subtilis and its relationship to development of actinomycin resistance. Biochem J 113, 29-37.

Van Dyk, T. K., Gatenby, A. A. \& LaRossa, R. A. (1989). Demonstration by genetic suppression of interaction of GroE products with many proteins. Nature 342, 451-453.

Zhang, Y. \& Cronan, J. E., Jr (1996). Polar allele duplication for transcriptional analysis of consecutive essential genes: application to a cluster of Escherichia coli fatty acid biosynthetic genes. J Bacteriol 178, 3614-3620.

Edited by: Tarek Msadek 